\title{
BLOW-UP CRITERION FOR COMPRESSIBLE VISCO-ELASTICITY EQUATIONS IN THREE DIMENSIONAL SPACE*
}

\author{
YI DU ${ }^{\dagger}$, CHUN LIU ${ }^{\ddagger}$, AND QINGTIAN ZHANG§
}

\begin{abstract}
In this paper, we prove a blow-up criterion for 3D compressible visco-elasticity in terms of the upper bound of the density and the deformation tensor. Due to the special structure of the equation, we get the cancellation to the derivatives of the density and transform tensor, which brings us the desired results.
\end{abstract}

Key words. 3D visco-elasticity, compressible, blow-up criterion.

AMS subject classifications. 35Q35, 76A10.

\section{Introduction}

Visco-elastic fluids exhibit the characteristics for both fluid and solid. The elastic behavior of these materials is attributed to the underlying microstructure or configurations, the deformation of the structures will cause the exchange of kinetic energy and elastic internal energy. The energy exchange is realized through a coupling of the transport of the internal elastic variables and the induced elastic stress.

To illustrate this coupled interaction, we denote $x(t, X)$ as the Eulerian coordinate, where $X$ is the Lagrangian coordinate of the particles. The velocity field defined on the Eulerian coordinate is

$$
u(t, x)=x_{t}(t, X(t, x))
$$

and the deformation tensor is

$$
F(t, x)=H(t, X(t, x))=\frac{\partial x}{\partial X}(t, X),
$$

which is determined by the transport equation (see $[3,8,16])$

$$
F_{t}+u \cdot \nabla F=\nabla u \cdot F
$$

This equation is regarded as the compatibility condition between the velocity $u$ and the deformation tensor $F$. For homogenous, hyper-elastic and isotropic material, the action function is (see $[11,16,17])$

$$
A(x)=\int_{0}^{T} \int_{\Omega_{0}} \frac{1}{2} \rho_{0}(X)\left|x_{t}(t, X)\right|^{2}-E(H)-\rho_{0}(X) \omega(\rho(x(X, t), t)) d X d t
$$

where $\rho_{0}(X)$ is the density in the undeformed configuration and will be chosen to be 1 in the following, $\rho$ is the density in the deformed configuration, $\Omega_{0}$ is the original

\footnotetext{
* Received: November 13, 2012; accepted (in revised form): March 25, 2013. Communicated by Yaguang Wang.

Yi Du was partly supported by the NSFC (Grant No. 11001088), and the Guang-Zhou Pearl River New-Star 2012J2200016. Chun Liu and Qingtian Zhang were partly supported by the NSF, (Grant DMS-1109107).

${ }^{\dagger}$ Department of Mathematics, South China Normal University, Guangzhou 510631, P.R. China (duyidy@gmail.com).

${ }^{\ddagger}$ Department of Mathematics, Penn. State University, USA (liu@math.psu.edu).

$\S$ Department of Mathematics, Penn. State University, USA (qingtian.zh@gmail.com).
} 
domain occupied by the material, $E(H)$ is the tangential elastic energy function, and $\omega(\rho)=C_{0} \frac{\gamma}{\gamma-1} \rho^{\gamma-1}$ is the normal elastic energy function, with the constant $C_{0}>0$. Without loss of generality, we take $C_{0}=1$. Taking a variation of $A(x)$ with respect to the flow map $x(t, X)$, we get the inviscid momentum equation in Eulerian coordinates $([10])$

$$
\rho\left(u_{t}+u \cdot \nabla u\right)+\nabla P=\nabla \cdot\left(\frac{1}{\operatorname{det} F} \frac{\partial E(F)}{\partial F} F^{T}\right),
$$

where $P(\rho)=\rho^{\gamma}$ is the hydrostatic pressure with the adiabatic index $\gamma>1, F^{T}$ is the transpose of $\mathrm{F}$, and the term $\frac{1}{\operatorname{det} F} \frac{\partial E(F)}{\partial F} F^{T}$ is the Cauchy-Green tensor. When we take the dissipation energy into consideration (see $[1,2]$ ), we can get the corresponding viscosity momentum equation

$$
\rho\left(u_{t}+u \cdot \nabla u\right)+\nabla P=\mu \Delta u+(\mu+\lambda) \nabla \operatorname{div} u+\nabla \cdot\left(\frac{1}{\operatorname{det} F} \frac{\partial E(F)}{\partial F} F^{T}\right) .
$$

Combining (1.3), (1.6), and the mass conservation equation with Hookean elasticity, we get the following viscoelasticity system:

$$
\left\{\begin{array}{l}
\rho_{t}+\nabla \cdot(\rho u)=0, \\
\rho\left(u_{t}+u \cdot \nabla u\right)+\nabla P=\mu \Delta u+(\mu+\lambda) \nabla \operatorname{div} u+\nabla \cdot\left(\frac{1}{\operatorname{det} F} F F^{T}\right), \\
F_{t}+u \cdot \nabla F=\nabla u F .
\end{array}\right.
$$

The viscosity coefficients $\mu$ and $\lambda$ are constants satisfying $\mu \geq 0,3 \lambda+2 \mu \geq 0$. In this paper, we consider the case $\rho_{0} \equiv 1$, under which equation (1.7) can be written as

$$
\left\{\begin{array}{l}
\rho_{t}+\nabla \cdot(\rho u)=0 \\
\rho\left(u_{t}+u \cdot \nabla u\right)+\nabla P=\mu \Delta u+(\mu+\lambda) \nabla \operatorname{div} u+\nabla \cdot\left(\rho F F^{T}\right), \\
F_{t}+u \cdot \nabla F=\nabla u F
\end{array}\right.
$$

The system (1.8), when $F$ does not exist, is the compressible Navier-Stokes equations. The global strong solutions existence for large initial data is still open. Therefore, there have been many efforts to study the blow-up criteria; see [7, 19] and references therein.

When we take the deformation tensor $F$ into consideration, the system (1.8) will be much more complicated, although the deformation tensor admits a transport equation which is similar to the continuity equation. The incompressible visco-elasticity has been studied by $[9,10,11,12,14,16,17]$, etc. Precisely, in 2D, the local strong solutions to large data and global strong solutions to small data have been studied in $[10,11,12,14,16]$. The global strong solutions in $3 \mathrm{D}$ with small data have been presented in [12]. Subsequently, the blow-up criteria for 2D Oldroyd model have been presented in [13], and a blow-up criterion in $3 \mathrm{D}$ with partial viscosity was proved in $[15]$.

For the compressible case, The local strong solutions with large data and global strong solutions with small data to (1.8) were proved in [4,5] and [18], respectively. Subsequently, Hu and Wang [6] presented a blow-up criterion for local strong solutions as $\|\nabla u\|_{L^{1}\left(0, T, L^{\infty}\left(\mathbb{R}^{3}\right)\right)}<\infty$. To proceed, we firstly introduce the following notation:

$$
D^{k}\left(\mathbb{R}^{3}\right)=\left\{u \in L_{l o c}^{1}\left(\mathbb{R}^{3}\right):\left\|\nabla^{k} u\right\|_{L^{2}\left(\mathbb{R}^{3}\right)}<\infty\right\},
$$




$$
D_{0}^{k}\left(\mathbb{R}^{3}\right)=\left\{u \in L^{6}\left(\mathbb{R}^{3}\right):\left\|\nabla^{k} u\right\|_{L^{2}\left(\mathbb{R}^{3}\right)}<\infty\right\} .
$$

$\operatorname{In}[6], \mathrm{Hu}$ and Wang proved the following results.

Theorem 1.1 (Hu-Wang [6]). Assume that the initial data satisfy $0 \leq \rho_{0} \in$ $H^{3}\left(\mathbb{R}^{3}\right), u_{0} \in D_{0}^{1}\left(\mathbb{R}^{3}\right) \cap D^{3}\left(\mathbb{R}^{3}\right), F_{0} \in H^{3}\left(\mathbb{R}^{3}\right), \nabla \cdot\left(\rho_{0} F_{0}\right)=0$, and

$$
-\mu \Delta u_{0}-(\lambda+\mu) \nabla \operatorname{divu}_{0}+A \nabla \rho_{0}^{\gamma}=\rho_{0} g,
$$

for some $g \in H^{1}\left(\mathbb{R}^{3}\right)$ with $\sqrt{\rho_{0}} g \in L^{2}\left(\mathbb{R}^{3}\right)$. There exist classical solutions $(\rho, u, F)$ to (1.8) satisfying

$$
\left\{\begin{array}{l}
(\rho, F) \in C\left(\left[0, T^{\star}\right), H^{3}\left(\mathbb{R}^{3}\right)\right), \\
u \in C\left(\left[0, T^{\star}\right), D_{0}^{1}\left(\mathbb{R}^{3}\right) \cap D^{3}\left(\mathbb{R}^{3}\right)\right) \cap L^{2}\left(\left[0, T^{\star}\right), D^{4}\left(\mathbb{R}^{3}\right)\right), \\
u_{t} \in L^{\infty}\left(\left[0, T^{\star}\right), D_{0}^{1}\left(\mathbb{R}^{3}\right)\right) \cap L^{2}\left(\left[0, T^{\star}\right), D^{2}\left(\mathbb{R}^{3}\right)\right), \\
\sqrt{\rho} u_{t} \in L^{\infty}\left(\left[0, T^{\star}\right), L^{2}\left(\mathbb{R}^{3}\right)\right),
\end{array}\right.
$$

where $T^{\star}$ is the maximal existence time. If $T^{\star}<\infty$ and $7 \mu>\lambda$, then

$$
\lim _{T \rightarrow T^{\star}} \int_{0}^{T}\|\nabla u\|_{L^{\infty}\left(\mathbb{R}^{3}\right)} d t=\infty .
$$

In fact, the result also holds on the torus $\mathbb{T}^{3}$. Enlightened by the work of Sun, Wang, and Zhang [19], in which the authors presented the blow-up criterion for a compressible Navier-Stokes system in terms of $\|\rho\|_{L^{\infty}\left(0, T, L^{\infty}\left(\mathbb{R}^{3}\right)\right)}$, we will establish a blow-up criterion in terms of the upper bounds of the density and the deformation tensor for the local strong solution to the $3 \mathrm{D}$ compressible visco-elasticity system. Here, we consider the system on the torus $\mathbb{T}^{3}$.

TheOREM 1.2. Assume that $(\rho, u, F)$ is the local strong solution of $(1.8)$ is as mentioned in (1.9), $\mu$, and $\lambda$ are as in Theorem 1.1, the initial data $\rho_{0} \equiv 1, F_{0} \equiv I$ (the identity matrix) and $u_{0}$ are as in Theorem 1.1, and $T^{\star}$ is the maximal existence time of the solution. If $T^{\star}<\infty$, then we have

$$
\lim _{T \uparrow T^{\star}} \sup \left\{\|\rho(t)\|_{L^{\infty}\left(0, T ; L^{\infty}\left(\mathbb{T}^{3}\right)\right)}+\|F\|_{L^{\infty}\left(0, T ; L^{\infty}\left(\mathbb{T}^{3}\right)\right)}\right\}=\infty .
$$

REMARK 1.1. With a slight modification of our proof, we can also get the same result for $\mathbb{R}^{3}$. In fact, in this paper we need to prove the results with the lower-bound for $\rho^{-1}$, which will introduce some difficulty into our proof. Here, we proved the results on $\mathbb{T}^{3}$ for simplicity.

If $\|\rho(t)\|_{L^{\infty}\left(0, T ; L^{\infty}\left(\mathbb{T}^{3}\right)\right)}$ and $\|F\|_{L^{\infty}\left(0, T ; L^{\infty}\left(\mathbb{T}^{3}\right)\right)}$ are bounded, the we can obtain a high integrability of velocity, which can be used to control the nonlinear term (see Lemma 3.2). The difficulty is to control the density and deformation, which satisfy transport equations. To do this, one needs the velocity bounded in $L^{1}\left(0, T ; W^{1, \infty}\left(\mathbb{T}^{3}\right)\right)$. On the other hand, we have to obtain some a priori bounds for $\nabla \rho$ and $\nabla F$ to prove $u \in L^{1}\left(0, T ; W^{1, \infty}\left(\mathbb{T}^{3}\right)\right)$, and the elasticity term $\nabla \cdot\left(\frac{1}{\operatorname{det} F} F F^{T}\right)$ in the momentum equation will bring extra difficulty to us. Thanks to the structure of the equations, we get the cancellation to the derivatives of $\rho$ and $F$ during our computation, which brings us the desired result. Moreover, we get the result without 
the restriction on $\rho^{-1}$, which is an advantage compared with the results of Sun, Wang, and Zhang [20].

In this paper, we follow the line of Sun, Wang, and Zhang [19, 20], and write

$$
L \triangleq \mu \Delta+(\lambda+\mu) \nabla \operatorname{div}
$$

in this case, the Lamé operator $L$ is elliptic.

Generally speaking, visco-elastic fluid is driven by three kinds of forces which present different mechanisms. They are the gradient of pressure, elastic deformation, and the inertial force. According to these mechanisms, we can decompose the velocity field into several parts. Here, since the pressure term and the elasticity term have similar form in the equation, we put them together and decompose the velocity field into two parts, that is

$$
u=v+w
$$

with

$$
L v=\nabla P-\nabla \cdot\left(\frac{1}{\operatorname{det} F} F F^{T}\right)
$$

and

$$
L w=\rho \dot{u},
$$

where

$$
\dot{u}=\partial_{t} u+u \cdot \nabla u
$$

By the decomposition, in order to obtain the regularity of $u$, it is sufficient to consider the regularity of $v$ and $w$, which satisfy the above elliptic equations.

Our paper is organized as follows. In Section 2, we shall present some preliminaries. In Section 3 we present some a priori estimates. The proof of Theorem 1.2 is presented in Section 4.

\section{Preliminaries}

Consider the following problem for the Lamé operator $L$ :

$$
\left\{\begin{array}{l}
\mu \triangle U+(\mu+\lambda) \nabla \operatorname{div} U=F, \quad \text { in } \mathbb{T}^{3}, \\
\left.U(x)\right|_{\partial \mathbb{T}^{3}}=0 .
\end{array}\right.
$$

The following lemmas were presented in $[19,20]$.

Lemma 2.1. Let $q \in(1, \infty)$ and $U$ be a solution of (2.1). There exists a constant $C$ depending only on $\lambda, \mu$, and $q$, such that the following estimates hold:

(1) If $F \in L^{q}\left(\mathbb{T}^{3}\right)$, then

$$
\|U\|_{W^{2, q}\left(\mathbb{T}^{3}\right)} \leq C\|F\|_{L^{q}\left(\mathbb{T}^{3}\right)},
$$

(2) If $F \in W^{-1, q}\left(\mathbb{T}^{3}\right)$ (i.e., $F=\operatorname{div} f$ with $\left.f=\left(f_{i j}\right)_{3 \times 3}, f_{i j} \in L^{q}\left(\mathbb{T}^{3}\right)\right)$, then

$$
\|U\|_{W^{1, q}\left(\mathbb{T}^{3}\right)} \leq C\|f\|_{L^{q}\left(\mathbb{T}^{3}\right)},
$$

(3) If $F=\operatorname{div} f$ with $f_{i j}=\partial_{k} H_{i j}^{k}$ and $h_{i j}^{k} \in W_{0}^{1, q}\left(\mathbb{T}^{3}\right)$ for $i, j, k=1,2,3$, then

$$
\|U\|_{L^{q}\left(\mathbb{T}^{3}\right)} \leq C\|h\|_{L^{q}\left(\mathbb{T}^{3}\right)} .
$$


Lemma 2.2. If $F=\operatorname{div} f$ with $f=\left(f_{i j}\right)_{3 \times 3}, \quad f_{i j} \in L^{\infty}\left(\mathbb{T}^{3}\right) \bigcap L^{2}\left(\mathbb{T}^{3}\right)$, then $\nabla U \in$ $B M O\left(\mathbb{T}^{3}\right)$ and there exists a constant $C$ depending only on $\lambda$ and $\mu$ such that

$$
\|\nabla U\|_{B M O\left(\mathbb{T}^{3}\right)} \leq C\left(\|f\|_{L^{\infty}\left(\mathbb{T}^{3}\right)}+\|f\|_{L^{2}\left(\mathbb{T}^{3}\right)}\right) .
$$

Lemma 2.3. Let $f \in W^{1, q}\left(\mathbb{T}^{3}\right)$ with $q \in(3, \infty)$. There exists a constant $C$ depending on $q$, such that

$$
\|f\|_{L^{\infty}\left(\mathbb{T}^{3}\right)} \leq C\left(1+\|f\|_{B M O\left(\mathbb{T}^{3}\right)}\right) \ln \left(e+\|\nabla f\|_{L^{q}\left(\mathbb{T}^{3}\right)}\right) .
$$

In the following, we use the notation, for matrices $(A)_{3 \times 3}$ and $(B)_{3 \times 3}$,

$$
A: B=A_{i j} B_{i j},
$$

where summation is applied to terms with repeated index.

\section{A priori estimate}

By the standard energy estimates, we have

LEMMA 3.1 (A priori estimate).

$$
\begin{gathered}
\|\rho(t)\|_{L^{1}\left(\mathbb{T}^{3}\right)}=\left\|\rho_{0}\right\|_{L^{1}\left(\mathbb{T}^{3}\right)}=\mathbb{M}\left(\mathbb{T}^{3}\right), \\
\|\sqrt{\rho} F\|_{L^{2}\left(\mathbb{T}^{3}\right)}+\|\rho(t)\|_{L^{\gamma}\left(\mathbb{T}^{3}\right)}^{\gamma}+\left\|\rho|u|^{2}(t)\right\|_{L^{1}\left(\mathbb{T}^{3}\right)}+\|\nabla u\|_{L^{2}\left((0, t) \times \mathbb{T}^{3}\right)}^{2} \\
\leq \mathbb{M}\left(\mathbb{T}^{3}\right)+C\left(\left\|\rho_{0}\left|u_{0}\right|^{2}\right\|_{L^{1}\left(\mathbb{T}^{3}\right)}+\left\|F_{0}\right\|_{L^{2}\left(\mathbb{T}^{3}\right)}\right),
\end{gathered}
$$

where the constant $\mathbb{M}\left(\mathbb{T}^{3}\right)$ is the measure of $\mathbb{T}^{3}$.

Lemma 3.2. Assume initial data as in Theorem 1.2, $2 \mu>\lambda$, and that the density $\rho$ and deformation tensor $F$ satisfy

$$
\|\rho\|_{L^{\infty}\left(0, T ; L^{\infty}\left(\mathbb{T}^{3}\right)\right)}+\|F\|_{L^{\infty}\left(0, T ; L^{\infty}\left(\mathbb{T}^{3}\right)\right)}<\infty .
$$

Then there exists $r \in(3,6)$ such that $\rho|u|^{r} \in L^{\infty}\left(0, T ; L^{1}\left(\mathbb{T}^{3}\right)\right)$, with

$$
\left\|\rho|u|^{r}\right\|_{L^{\infty}\left(0, T ; L^{1}\left(\mathbb{T}^{3}\right)\right)} \leq C .
$$

Here $C$ depends on $T,\|\rho\|_{L^{\infty}\left(\mathbb{T}^{3}\right)},\|F\|_{L^{\infty}\left(\mathbb{T}^{3}\right)}$, and the initial data.

Proof. Multiplying the equation $(1.8)_{2}$ by $r|u|^{r-2} u$ and integrating the resulting equation on $\mathbb{T}^{3}$, we obtain

$$
\begin{aligned}
& \quad \frac{d}{d t} \int_{\mathbb{T}^{3}} \rho|u|^{r} d x+\int_{\mathbb{T}^{3}} r|u|^{r-2}\left(\mu|\nabla u|^{2}+(\lambda+\mu)(\operatorname{div} u)^{2}\right) \\
& \quad+r(r-2)\left(\mu|u|^{r-2}|\nabla| u||^{2}+(\lambda+\mu) \operatorname{div} u|u|^{r-3} u \cdot \nabla|u|\right) d x \\
& =\int_{\mathbb{T}^{3}} r P(\rho) \operatorname{div}\left(|u|^{r-2} u\right) d x-\int_{\mathbb{T}^{3}} r \nabla\left(|u|^{r-2} u\right):\left(\rho F F^{T}\right) d x .
\end{aligned}
$$

By using the fact that $|\nabla u| \geq|\nabla| u||$, the term in second integrand can be estimated by

$$
r|u|^{r-2}\left[\mu|\nabla u|^{2}+(\lambda+\mu)(\operatorname{div} u)^{2}+(r-2) \mu|\nabla| u||^{2}-(\lambda+\mu)(r-2)|\nabla| u|| \operatorname{div} u\right]
$$




$$
\begin{aligned}
& \geq r|u|^{r-2}\left[\mu|\nabla u|^{2}+\left.(r-2)\left(\mu-(\lambda+\mu) \frac{r-2}{4}\right)|\nabla| u\right|^{2}\right] \\
& \geq C|u|^{r-2}|\nabla u|^{2} .
\end{aligned}
$$

For the pressure term $P(\rho)=\rho^{\gamma}$ with $\gamma>1$, recalling Assumption (3.2), we have

$$
\begin{aligned}
\int_{\mathbb{T}^{3}} P(\rho) \operatorname{div}\left(|u|^{r-2} u\right) d x & \leq C \int_{\mathbb{T}^{3}} P(\rho)|u|^{r-2}|\nabla u| d x \leq C \int_{\mathbb{T}^{3}} \rho^{\frac{1}{2}}|u|^{r-2}|\nabla u| d x \\
& \leq \epsilon \int_{\mathbb{T}^{3}}|u|^{r-2}|\nabla u|^{2} d x+\frac{C}{\epsilon} \int_{\mathbb{T}^{3}} \rho^{\frac{r-2}{r}}|u|^{r-2} \rho^{\frac{2}{r}} d x \\
& \leq \epsilon \int_{\mathbb{T}^{3}}|u|^{r-2}|\nabla u|^{2} d x+\frac{C}{\epsilon}\left(\int_{\mathbb{T}^{3}} \rho|u|^{r} d x\right)^{\frac{r-2}{r}} \cdot \int_{\mathbb{T}^{3}} \rho d x .
\end{aligned}
$$

By Lemma 3.1, $\|\rho\|_{L^{1}\left(\mathbb{T}^{3}\right)}=\left\|\rho_{0}\right\|_{L^{1}\left(\mathbb{T}^{3}\right)}$, we then get

$$
\int_{\mathbb{T}^{3}} P(\rho) \operatorname{div}\left(|u|^{r-2} u\right) d x \leq \epsilon \int_{\mathbb{T}^{3}}|u|^{r-2}|\nabla u|^{2} d x+\frac{C}{\epsilon}\left(\int_{\mathbb{T}^{3}} \rho|u|^{r} d x\right)^{\frac{r-2}{r}} .
$$

Similarly, for the elasticity term, recalling $\rho_{0} \geq \epsilon_{0}>0$, Lemma 2.4, and (3.2), we have

$$
\begin{aligned}
\int_{\mathbb{T}^{3}}\left|\nabla\left(|u|^{r-2} u\right): \rho F F^{T}\right| d x & \leq C \int_{\mathbb{T}^{3}} \rho|u|^{r-2}|\nabla u| d x \\
& \leq \epsilon \int_{\mathbb{T}^{3}}|u|^{r-2}|\nabla u|^{2} d x+\frac{C}{\epsilon}\left(\int_{\mathbb{T}^{3}} \rho|u|^{r} d x\right)^{\frac{r-2}{r}} .
\end{aligned}
$$

Then, (3.4)-(3.8) imply the desired estimate.

Proposition 3.3. Under Assumption (3.2), we have

$$
\|\nabla w\|_{L^{\infty}\left(0, T, L^{2}\left(\mathbb{T}^{3}\right)\right)},\left\|\rho^{\frac{1}{2}} \partial_{t} w\right\|_{L^{2}\left(0, T, L^{2}\left(\mathbb{T}^{3}\right)\right)},\left\|\nabla^{2} w\right\|_{L^{2}\left(0, T, L^{2}\left(\mathbb{T}^{3}\right)\right)} \leq C,
$$

where $C$ depends on $T,\|\rho\|_{L^{\infty}\left(0, T, L^{\infty}\left(\mathbb{T}^{3}\right)\right)},\|F\|_{L^{\infty}\left(0, T, L^{\infty}\left(\mathbb{T}^{3}\right)\right)}$, and the initial data.

Proof. Using the momentum equation, we get

$$
\left\{\begin{array}{l}
\rho \partial_{t} w-L w=\rho G, \quad \text { in }[0, T) \times \mathbb{T}^{3}, \\
w(0, x)=w_{0}(x),
\end{array}\right.
$$

where

$$
\begin{aligned}
G=- & u \operatorname{div} u-L^{-1} \nabla\left(\partial_{t} P\right)+L^{-1} \nabla \partial_{t}\left(\rho F F^{T}\right) \\
=- & u \operatorname{div} u+L^{-1} \nabla \operatorname{div}(P u)+L^{-1} \nabla\left[\left(\rho P^{\prime}(\rho)-P\right) \operatorname{div} u\right] \\
& +L^{-1} \nabla\left[\nabla u \cdot\left(\rho F F^{T}\right)+\rho F F^{T}(\nabla u)^{T}-\operatorname{div}\left(u \otimes \rho F F^{T}\right)\right] .
\end{aligned}
$$

Multiplying the equation with $\partial_{t} w$ and integrating over $\mathbb{T}^{3}$, with the Hölder inequality, we get

$$
\frac{d}{d t} \int_{\mathbb{T}^{3}} \mu|\nabla w|^{2}+(\lambda+\mu)|\operatorname{div} w|^{2} d x+\frac{1}{2} \int_{\mathbb{T}^{3}} \rho\left|\partial_{t} w\right|^{2} d x \leq \frac{1}{2}\|\sqrt{\rho} G\|_{L^{2}\left(\mathbb{T}^{3}\right)}^{2} .
$$

Now, we shall estimate $\|\sqrt{\rho} G\|_{L^{2}\left(\mathbb{T}^{3}\right)}^{2}$ term by term. First,

$$
\|\sqrt{\rho} u \operatorname{div} u\|_{L^{2}\left(\mathbb{T}^{3}\right)} \leq C\left\|\rho^{\frac{1}{r}} u\right\|_{L^{r}\left(\mathbb{T}^{3}\right)}\|\nabla u\|_{L^{\frac{2 r}{r-2}}\left(\mathbb{T}^{3}\right)}
$$




$$
\leq C(\epsilon)\|\nabla w\|_{L^{2}\left(\mathbb{T}^{3}\right)}+\epsilon\left\|\nabla^{2} w\right\|_{L^{2}\left(\mathbb{T}^{3}\right)}+C,
$$

where $2 \leq r<6$, and we used the interpolation inequality

$$
\|\cdot\|_{L^{r}\left(\mathbb{T}^{3}\right)} \leq C(\epsilon)\|\cdot\|_{L^{2}\left(\mathbb{T}^{3}\right)}+\epsilon\|\nabla \cdot\|_{L^{2}\left(\mathbb{T}^{3}\right)} .
$$

From the estimates for Lamé operator and the energy estimates in Lemma 3.1, we have

$$
\left\|\sqrt{\rho} L^{-1} \nabla \operatorname{div}(P u)\right\|_{L^{2}\left(\mathbb{T}^{3}\right)} \leq C\|P u\|_{L^{2}\left(\mathbb{T}^{3}\right)} \leq C\|\sqrt{\rho} u\|_{L^{2}\left(\mathbb{T}^{3}\right)} \leq C,
$$

and

$$
\begin{aligned}
& \quad\left\|\sqrt{\rho} L^{-1} \nabla\left[\nabla u \cdot\left(\rho F F^{T}\right)+\rho F F^{T}(\nabla u)^{T}-\nabla \cdot\left(u \otimes \rho F F^{T}\right)\right]\right\|_{L^{2}\left(\mathbb{T}^{3}\right)} \\
& \leq C\|\sqrt{\rho}\|_{L^{3}\left(\mathbb{T}^{3}\right)}\left\|L^{-1} \nabla\left[\nabla u \cdot\left(\rho F F^{T}\right)+\rho F F^{T}(\nabla u)^{T}\right]\right\|_{L^{6}\left(\mathbb{T}^{3}\right)} \\
& \quad+C\left\|L^{-1} \nabla\left[\nabla \cdot\left(u \otimes \rho F F^{T}\right)\right]\right\|_{L^{2}\left(\mathbb{T}^{3}\right)} \\
& \leq C\|\nabla u\|_{L^{2}\left(\mathbb{T}^{3}\right)}+C .
\end{aligned}
$$

Similarly, we have

$$
\left\|\sqrt{\rho} L^{-1} \nabla\left[\left(\rho P^{\prime}-P\right) \operatorname{div} u\right]\right\|_{L^{2}\left(\mathbb{T}^{3}\right)} \leq C\|\nabla u\|_{L^{2}\left(\mathbb{T}^{3}\right)} .
$$

Then, the results (3.9) follow from (3.11)-(3.17), Lemma 2.1, and Lemma 3.1.

COROLlary 3.1. Under the assumption of Lemma 3.2, we have

$$
\|\nabla u\|_{L^{\infty}\left(0, T, L^{2}\left(\mathbb{T}^{3}\right)\right)},\|\nabla u\|_{L^{2}\left(0, T, L^{q}\left(\mathbb{T}^{3}\right)\right)} \leq C,
$$

for any $q \in[2,6]$.

\section{Proof of Theorem 1.2}

Proposition 4.1. Suppose $T^{\star}<\infty$ is the maximal existence time. Then for all $T \in\left[0 . T^{\star}\right)$ and $3<q<6$, if

$$
\left\|\rho, \rho^{-1}\right\|_{L^{\infty}\left(0, T, L^{\infty}\left(\mathbb{T}^{3}\right)\right)}+\|F\|_{L^{\infty}\left(0, T, L^{\infty}\left(\mathbb{T}^{3}\right)\right)}<\infty,
$$

then we obtain

$$
\left\|\nabla^{2} w\right\|_{L^{2}\left(0, T ; L^{q}\left(\mathbb{T}^{3}\right)\right)} \leq C
$$

and

$$
\int_{0}^{T}\|\nabla u\|_{L^{\infty}\left(\mathbb{T}^{3}\right)} d s<\infty
$$
have

Proof. We first estimate $\left\|\nabla^{2} w\right\|_{L^{2}\left(0, T ; L^{q}\left(\mathbb{T}^{3}\right)\right)}$. By Lemma 2.1 and (1.15), we

$$
\left\|\nabla^{2} w\right\|_{L^{q}\left(\mathbb{T}^{3}\right)} \leq\|\rho \dot{u}\|_{L^{q}\left(\mathbb{T}^{3}\right)} .
$$

Noting that

$$
\rho \dot{u}-L u+\nabla P=\nabla \cdot\left(\frac{1}{\operatorname{det} F} F F^{T}\right)
$$


and taking $\partial_{t}$ of this equation, we get

$$
\rho_{t} \dot{u}+\rho \dot{u}_{t}-L u_{t}+\nabla P_{t}=\nabla \cdot\left(\frac{1}{\operatorname{det} F} F F^{T}\right)_{t} .
$$

Applying $u \otimes$ and divergence to (4.5), we have

$$
\nabla \cdot(\rho u) \dot{u}+\rho u \cdot \nabla \dot{u}-\nabla \cdot(u \otimes L u)+\nabla \cdot(u \otimes \nabla P)=\nabla \cdot\left[u \otimes \nabla \cdot\left(\frac{1}{\operatorname{det} F} F F^{T}\right)\right] .
$$

Adding (4.6) and (4.7) together, we get

$$
\begin{aligned}
\rho \dot{u}_{t}+\rho u \cdot \nabla \dot{u}+\nabla P_{t} & +\nabla \cdot[u \otimes \nabla P] \\
=\mu\left[\triangle u_{t}+\nabla \cdot(u \otimes \triangle u)\right] & +(\lambda+\mu)\left[\nabla \operatorname{div} u_{t}+\operatorname{div}(u \otimes \nabla \operatorname{div} u)\right] \\
& +\nabla \cdot\left[\left(\frac{1}{\operatorname{det} F} F F^{T}\right)_{t}+u \otimes \nabla \cdot\left(\frac{1}{\operatorname{det} F} F F^{T}\right)\right] .
\end{aligned}
$$

Multiplying the above equation by $\dot{u}$ and integrating over $\mathbb{T}^{3}$, we get

$$
\begin{aligned}
& \frac{d}{d t} \int_{\mathbb{T}^{3}} \frac{1}{2} \rho|\dot{u}|^{2} d x-\mu \int_{\mathbb{T}^{3}}\left(\Delta u_{t}+\operatorname{div}(u \otimes \triangle u)\right) \cdot \dot{u} d x-(\lambda+\mu) \int_{\mathbb{T}^{3}}\left(\nabla \operatorname{div} u_{t}+\operatorname{div}(u \otimes \nabla \operatorname{div} u)\right) \cdot \dot{u} d x \\
&=\int_{\mathbb{T}^{3}} P_{t} \operatorname{div} \dot{u} d x+\int_{\mathbb{T}^{3}} u \cdot \nabla \dot{u} \cdot \nabla P d x \\
& \quad+\int_{\mathbb{T}^{3}} \nabla \cdot\left[\left(\frac{1}{\operatorname{det} F} F F^{T}\right)_{t}+u \otimes \nabla \cdot\left(\frac{1}{\operatorname{det} F} F F^{T}\right)\right] \cdot \dot{u} d x .
\end{aligned}
$$

The estimates of the second and the third terms on the left hand side, as well as the first and the second terms on the right hand side in (4.9), are the same as in [19, 20]. For completeness, we give a brief proof as follows:

$$
\begin{aligned}
& -\int_{\mathbb{T}^{3}}\left(\triangle u_{t}+\operatorname{div}(u \otimes \Delta u)\right) \cdot \dot{u} d x \\
= & \int_{\mathbb{T}^{3}} \nabla u_{t}: \nabla \dot{u}+u \otimes \Delta u: \nabla \dot{u} d x \\
= & \int_{\mathbb{T}^{3}}|\nabla \dot{u}|^{2}-\nabla(u \cdot \nabla u): \nabla \dot{u}+u \otimes \Delta u: \nabla \dot{u} d x \\
= & \int_{\mathbb{T}^{3}}\left[|\nabla \dot{u}|^{2}-(\nabla u \nabla u): \nabla \dot{u}+((u \cdot \nabla) \nabla \dot{u}): \nabla u\right. \\
\geq & \int_{\mathbb{T}^{3}}\left[\frac{4}{5}|\nabla \dot{u}|^{2}-C|\nabla u|^{4}\right] d x,
\end{aligned}
$$

and

$$
\begin{gathered}
-\int_{\mathbb{T}^{3}}\left(\nabla \operatorname{div} u_{t}+\operatorname{div}(u \otimes \nabla \operatorname{div} u)\right) \cdot \dot{u} d x \\
=\int_{\mathbb{T}^{3}}\left[|\operatorname{div} \dot{u}|^{2}-\operatorname{div} \dot{u} \nabla u:(\nabla u)^{T}-\operatorname{div} u(\nabla \dot{u})^{T}: \nabla u+\operatorname{div} \dot{u}(\operatorname{div} u)^{2}\right] d x \\
\geq \int_{\mathbb{T}^{3}}\left[\frac{1}{2}|\operatorname{div} \dot{u}|^{2}-\frac{1}{10}|\nabla \dot{u}|^{2}-C|\nabla u|^{4}\right] d x .
\end{gathered}
$$


We continue to estimate the pressure term:

$$
\begin{aligned}
& \int_{\mathbb{T}^{3}} P_{t} \operatorname{div} \dot{u}+(u \cdot \nabla \dot{u}) \cdot \nabla P d x \\
= & \int_{\mathbb{T}^{3}}-\rho P^{\prime}(\rho) \operatorname{div} u \operatorname{div} \dot{u}+P\left[\operatorname{div} u \operatorname{div} \dot{u}-(\nabla u)^{T}:(\nabla \dot{u})\right] d x \\
\leq & C\|\nabla u\|_{L^{2}\left(\mathbb{T}^{3}\right)}\|\nabla \dot{u}\|_{L^{2}\left(\mathbb{T}^{3}\right)} \leq C\|\nabla \dot{u}\|_{L^{2}\left(\mathbb{T}^{3}\right)} .
\end{aligned}
$$

Next, we estimate the elasticity term $\int_{\mathbb{T}^{3}} \nabla \cdot\left[\left(\frac{1}{\operatorname{det} F} F F^{T}\right)_{t}+u \otimes \nabla \cdot\left(\frac{1}{\operatorname{det} F} F F^{T}\right)\right] \cdot$ $\dot{u} d x$. From (1.8), we get

$$
\begin{aligned}
& \int_{\mathbb{T}^{3}} \nabla \cdot\left[\left(\frac{1}{\operatorname{det} F} F F^{T}\right)_{t}+u \otimes \nabla \cdot\left(\frac{1}{\operatorname{det} F} F F^{T}\right)\right] \cdot \dot{u} d x \\
= & \int_{\mathbb{T}^{3}} \nabla \cdot\left[\nabla u \cdot\left(\frac{1}{\operatorname{det} F} F F^{T}\right)+\frac{1}{\operatorname{det} F} F F^{T}(\nabla u)^{T}-\frac{1}{\operatorname{det} F} F F^{T}(\nabla \cdot u)\right] \dot{u} d x \\
& +\int_{\mathbb{T}^{3}} \nabla \cdot\left[u \otimes \nabla \cdot\left(\frac{1}{\operatorname{det} F} F F^{T}\right)-u \cdot \nabla\left(\frac{1}{\operatorname{det} F} F F^{T}\right)\right] \dot{u} d x .
\end{aligned}
$$

Since $\|\rho\|_{L^{\infty}\left(0, T, L^{\infty}\left(\mathbb{T}^{3}\right)\right)},\|F\|_{L^{\infty}\left(0, T, L^{\infty}\left(\mathbb{T}^{3}\right)\right)}<\infty$, we get the bound for the first three terms on the right hand side of the above equation as follows:

$$
\left|\int_{\mathbb{T}^{3}} \nabla \cdot\left[\nabla u \cdot\left(\frac{1}{\operatorname{det} F} F F^{T}\right)+\frac{1}{\operatorname{det} F} F F^{T}\left(\nabla u^{T}-\nabla \cdot u\right)\right] \dot{u} d x\right| \leq C \int_{\mathbb{T}^{3}}|\nabla u||\nabla \dot{u}| d x .
$$

As to the last term on the right hand side of (4.13), we have a cancellation:

$$
\begin{aligned}
& \nabla \cdot\left[u \otimes \nabla \cdot\left(\frac{1}{\operatorname{det} F} F F^{T}\right)-u \cdot \nabla\left(\frac{1}{\operatorname{det} F} F F^{T}\right)\right] \cdot \dot{u} \\
= & -\partial_{i}\left[\partial_{j} u_{i}\left(\frac{1}{\operatorname{det} F} F_{j k} F_{l k}\right)\right] \dot{u}_{l}+\partial_{i}\left[\partial_{j} u_{j}\left(\frac{1}{\operatorname{det} F} F_{j k} F_{l k}\right)\right] \dot{u}_{l} .
\end{aligned}
$$

Therefore,

$$
\begin{aligned}
& \int_{\mathbb{T}^{3}} \nabla \cdot\left[u \otimes \nabla \cdot\left(\frac{1}{\operatorname{det} F} F F^{T}\right)-u \cdot \nabla\left(\frac{1}{\operatorname{det} F} F F^{T}\right)\right] \cdot \dot{u} d x \\
& \leq C\|\nabla \dot{u}\|_{L^{2}\left(\mathbb{T}^{3}\right)}\|\nabla u\|_{L^{2}\left(\mathbb{T}^{3}\right)} .
\end{aligned}
$$

Combining the above estimates, and recalling Lemma 3.1, we get

$$
\frac{d}{d t} \int_{\mathbb{T}^{3}} \rho|\dot{u}|^{2} d x+\int_{\mathbb{T}^{3}}|\nabla \dot{u}|^{2} d x \leq C\left(1+\|\nabla u\|_{L^{4}\left(\mathbb{T}^{3}\right)}^{4}\right) .
$$

Note that

$$
\begin{aligned}
\|\nabla u\|_{L^{4}\left(\mathbb{T}^{3}\right)}^{4} & \leq\|\nabla u\|_{L^{2}\left(\mathbb{T}^{3}\right)}\|\nabla u\|_{L^{6}\left(\mathbb{T}^{3}\right)}^{3} \\
& \leq C\|\nabla u\|_{L^{6}\left(\mathbb{T}^{3}\right)}^{2}\left(\|\nabla w\|_{L^{6}\left(\mathbb{T}^{3}\right)}+\|\nabla v\|_{L^{6}\left(\mathbb{T}^{3}\right)}\right) \\
& \leq C\|\nabla u\|_{L^{6}\left(\mathbb{T}^{3}\right)}^{2}\left(1+\|\dot{u}\|_{L^{2}\left(\mathbb{T}^{3}\right)}\right) .
\end{aligned}
$$

Substituting this estimate into (4.17) and using Corollary 3.1, we have $\|\nabla u(t)\|_{L^{6}\left(\mathbb{T}^{3}\right)}^{2} \in$ $L^{1}(0, T)$, and then conclude by Gronwall's inequality that

$$
\|\sqrt{\rho} \dot{u}\|_{L^{\infty}\left(0, T ; L^{2}\left(\mathbb{T}^{3}\right)\right)}+\|\nabla \dot{u}\|_{L^{2}\left((0, T) \times \mathbb{T}^{3}\right)} \leq C .
$$


Recalling (4.4) and Lemma 2.1, (4.2) is verified.

In the following, we shall prove (4.3). Note that

$$
\begin{aligned}
\|\nabla u\|_{L^{1}\left(0, T ; L^{\infty}\left(\mathbb{T}^{3}\right)\right)} & \leq C\|u\|_{L^{1}\left(0, T ; W^{2, q}\left(\mathbb{T}^{3}\right)\right)} \\
& \leq C\left(\|v\|_{L^{1}\left(0, T ; W^{2, q}\left(\mathbb{T}^{3}\right)\right)}+\|w\|_{L^{1}\left(0, T ; W^{2, q}\left(\mathbb{T}^{3}\right)\right)}\right),
\end{aligned}
$$

where $3<q<6$. By Lemma 2.1, $\|v\|_{W^{2, q}\left(\mathbb{T}^{3}\right)} \leq\|\nabla P\|_{L^{q}\left(\mathbb{T}^{3}\right)}+\left\|\nabla \cdot\left(\frac{1}{\operatorname{det} F} F F^{T}\right)\right\|_{L^{q}\left(\mathbb{T}^{3}\right)}$, and from the assumption (4.1) we have

$$
\|\nabla P\|_{L^{q}\left(\mathbb{T}^{3}\right)} \leq C\|\nabla \rho\|_{L^{q}\left(\mathbb{T}^{3}\right)},
$$

and

$$
\begin{aligned}
& \left\|\nabla \cdot\left(\frac{1}{\operatorname{det} F} F F^{T}\right)\right\|_{L^{q}\left(\mathbb{T}^{3}\right)} \leq\left\|\nabla \cdot\left(\rho F F^{T}\right)\right\|_{L^{q}\left(\mathbb{T}^{3}\right)} \\
\leq & C\|\nabla \rho\|_{L^{q}\left(\mathbb{T}^{3}\right)}\|F\|_{L^{\infty}\left(\mathbb{T}^{3}\right)}^{2}+C\|\rho\|_{L^{\infty}\left(\mathbb{T}^{3}\right)}\|\nabla F\|_{L^{q}\left(\mathbb{T}^{3}\right)}\|F\|_{L^{\infty}\left(\mathbb{T}^{3}\right)} \\
\leq & C\|\nabla \rho\|_{L^{q}\left(\mathbb{T}^{3}\right)}+C\|\nabla F\|_{L^{q}\left(\mathbb{T}^{3}\right)}
\end{aligned}
$$

where we used the fact that $\rho \cdot \operatorname{det} F=1$. Therefore, recalling (4.2), to bound $\|\nabla u\|_{L^{1}\left(0, T, L^{\infty}\left(\mathbb{T}^{3}\right)\right)}$, it suffices to bound $\|\nabla \rho\|_{L^{q}\left(\mathbb{T}^{3}\right)}$ and $\|\nabla F\|_{L^{q}\left(\mathbb{T}^{3}\right)}$. Taking the gradient of both sides of equation (1.8) $)_{1}$ and $(1.8)_{3}$, we have

$$
(\nabla \rho)_{t}+u \cdot \nabla(\nabla \rho)=-\nabla(\rho \nabla \cdot u)-\nabla u \cdot \nabla \rho,
$$

and

$$
\rho(\nabla F)_{t}+\rho u \cdot \nabla(\nabla F)=\rho \nabla(\nabla u F)-\rho \nabla u \nabla F .
$$

Multiplying (4.23) and (4.24) by $q \rho|\nabla \rho|^{q-2} \nabla \rho$ and $q \rho|\nabla F|^{q-2} \nabla F$, respectively, we have

$$
\begin{aligned}
\frac{d}{d t} \int_{\mathbb{T}^{3}} \rho|\nabla \rho|^{q} d x & =-q \int_{\mathbb{T}^{3}} \nabla(\rho \nabla \cdot u) \rho|\nabla \rho|^{q-2} \nabla \rho d x-q \int_{\mathbb{T}^{3}} \nabla u \cdot \nabla \rho \rho|\nabla \rho|^{q-2} \nabla \rho d x \\
& \leq C \int_{\mathbb{T}^{3}} \rho\left|\nabla u \left\|\left.\nabla \rho\right|^{q} d x+C \int_{\mathbb{T}^{3}} \rho\left|\nabla^{2} u \| \nabla \rho\right|^{q-1} d x\right.\right. \\
& \leq C\|\nabla u\|_{L^{\infty}\left(\mathbb{T}^{3}\right)}\left\|\rho^{1 / q} \nabla \rho\right\|_{L^{q}\left(\mathbb{T}^{3}\right)}^{q}+C\left\|\nabla^{2} u\right\|_{L^{q}\left(\mathbb{T}^{3}\right)}\left\|\rho^{1 / q} \nabla \rho\right\|_{L^{q}\left(\mathbb{T}^{3}\right)}^{q-1},
\end{aligned}
$$

as well as

$$
\begin{aligned}
\frac{d}{d t} \int_{\mathbb{T}^{3}} \rho|\nabla F|^{q} d x & \leq\left. C \int_{\mathbb{T}^{3}}|\rho \nabla(\nabla u F)| \nabla F\right|^{q-2} \nabla F\left|d x+C \int_{\mathbb{T}^{3}}\right| \rho \nabla u \nabla F|\nabla F|^{q-2} \nabla F \mid d x \\
& \leq C\left\|\nabla^{2} u\right\|_{L^{q}\left(\mathbb{T}^{3}\right)}\left\|\rho^{1 / q} \nabla F\right\|_{L^{q}\left(\mathbb{T}^{3}\right)}^{q-1}+C\|\nabla u\|_{L^{\infty}\left(\mathbb{T}^{3}\right)}\left\|\rho^{1 / q} \nabla F\right\|_{L^{q}\left(\mathbb{T}^{3}\right)^{\prime}}^{q}
\end{aligned}
$$

From (4.25) and (4.26), we get

$$
\begin{aligned}
& \quad \frac{d}{d t}\left(\left\|\rho^{1 / q} \nabla \rho\right\|_{L^{q}\left(\mathbb{T}^{3}\right)}^{q}+\left\|\rho^{1 / q} \nabla F\right\|_{L^{q}\left(\mathbb{T}^{3}\right)}^{q}\right) \\
& \leq C\left\|\nabla^{2} u\right\|_{L^{q}\left(\mathbb{T}^{3}\right)}\left(\left\|\rho^{1 / q} \nabla \rho\right\|_{L^{q}\left(\mathbb{T}^{3}\right)}^{q-1}+\left\|\rho^{1 / q} \nabla F\right\|_{L^{q}\left(\mathbb{T}^{3}\right)}^{q-1}\right) \\
& \quad+C\|\nabla u\|_{L^{\infty}\left(\mathbb{T}^{3}\right)}\left(\left\|\rho^{1 / q} \nabla \rho\right\|_{L^{q}\left(\mathbb{T}^{3}\right)}^{q}+\left\|\rho^{1 / q} \nabla F\right\|_{L^{q}\left(\mathbb{T}^{3}\right)}^{q}\right) .
\end{aligned}
$$


Noting that

$$
\begin{aligned}
& \|\nabla u\|_{L^{\infty}\left(\mathbb{T}^{3}\right)} \leq\|\nabla v\|_{L^{\infty}\left(\mathbb{T}^{3}\right)}+\|\nabla w\|_{L^{\infty}\left(\mathbb{T}^{3}\right)}, \\
& \left\|\nabla^{2} u\right\|_{L^{q}\left(\mathbb{T}^{3}\right)} \leq\left\|\nabla^{2} v\right\|_{L^{q}\left(\mathbb{T}^{3}\right)}+\left\|\nabla^{2} w\right\|_{L^{q}\left(\mathbb{T}^{3}\right)},
\end{aligned}
$$

and by Lemma 2.2 ,

$$
\begin{aligned}
\|\nabla v\|_{L^{\infty}\left(\mathbb{T}^{3}\right)} & \leq 1+\|\nabla v\|_{B M O\left(\mathbb{T}^{3}\right)} \ln \left(e+\left\|\nabla^{2} v\right\|_{L^{q}\left(\mathbb{T}^{3}\right)}\right) \\
& \leq 1+C \ln \left(e+\left\|\nabla^{2} v\right\|_{L^{q}\left(\mathbb{T}^{3}\right)}\right) .
\end{aligned}
$$

Then from (4.2), we obtain

$$
\begin{aligned}
& \frac{d}{d t}\left(e+\left\|\rho^{1 / q} \nabla \rho\right\|_{L^{q}\left(\mathbb{T}^{3}\right)}+\left\|\rho^{1 / q} \nabla F\right\|_{L^{q}\left(\mathbb{T}^{3}\right)}\right) \\
& \leq C\left[1+\ln \left(e+\left\|\rho^{1 / q} \nabla \rho\right\|_{L^{q}\left(\mathbb{T}^{3}\right)}+\left\|\rho^{1 / q} \nabla F\right\|_{L^{q}\left(\mathbb{T}^{3}\right)}\right)\right] \\
& \cdot\left(e+\left\|\rho^{1 / q} \nabla \rho\right\|_{L^{q}\left(\mathbb{T}^{3}\right)}+\left\|\rho^{1 / q} \nabla F\right\|_{L^{q}\left(\mathbb{T}^{3}\right)}\right) .
\end{aligned}
$$

Using Gronwall's inequality, we get that $\left\|\rho^{1 / q} \nabla \rho\right\|_{L^{q}\left(\mathbb{T}^{3}\right)}$ and $\left\|\rho^{1 / q} \nabla F\right\|_{L^{q}\left(\mathbb{T}^{3}\right)}$ are finite, and hence $\left\|\nabla^{2} v\right\|_{L^{q}\left(\mathbb{T}^{3}\right)}$ is bounded. Combining this with (4.2), (4.3) is verified.

Remark 4.1. Since $\rho^{-1}=\operatorname{det} F$, we have $\left\|\rho^{-1}\right\|_{L^{\infty}\left(\mathbb{T}^{3}\right)} \leq\|F\|_{L^{\infty}\left(\mathbb{T}^{3}\right)}^{3}<\infty$. Namely, if we restrict $\|F\|_{L^{\infty}\left(0, T, L^{\infty}\left(\mathbb{T}^{3}\right)\right)}<\infty$, then $\left\|\rho^{-1}\right\|_{L^{\infty}\left(0, T, L^{\infty}\left(\mathbb{T}^{3}\right)\right)}<\infty$ will hold automatically.

Now we are ready to prove our main theorem by contradiction.

Proof of Theorem 1.2. Suppose the maximal existence time of solution $T^{\star}<$ $\infty$, and $\|\rho\|_{L^{\infty}\left(0, T^{\star}, L^{\infty}\left(\mathbb{T}^{3}\right)\right)}+\|F\|_{L^{\infty}\left(0, T^{\star}, L^{\infty}\left(\mathbb{T}^{3}\right)\right)}<\infty$. By Remark 4.1 and Proposition 4.1 , we obtain

$$
\|\nabla u\|_{L^{1}\left(0, T^{\star}, L^{\infty}\left(\mathbb{T}^{3}\right)\right)}<\infty .
$$

This is a contradiction with Theorem 1.1.

Acknowledgment. The authors express their sincere appreciation to professor Zhen Lei for his constructive suggestion and discussion. They also thank Dr. Geng Chen for his kind help. The work proceeded substantially while the first author was visiting the Department of Mathematics, Penn State University. He thanks deeply the Department of Mathematics and professor Chun Liu for the warm hospitality.

\section{REFERENCES}

[1] J. Ericksen, Conservation laws for liquid crystals, Trans. Soc. Rheol., 5, 22-34, 1961.

[2] M. Doi and S.F. Edwards, The Theory of Polymer Dynamics, Oxford Science Publication, 1986.

[3] M.E. Gurtin, An Introduction to Continuum Mechanics, Mathematics in Science and Engineering, Academic Press, New York, London, 158, 1981.

[4] X. Hu and D. Wang, Local strong solution to the compressible viscoelastic flow with large data, J. Diff. Equ., 249, 1179-1198, 2010.

[5] X. Hu and D. Wang, Global existence for the multi-dimensional compressible viscoelastic flow, J. Diff. Equ., 250, 1200-1231, 2011. 
[6] X. Hu and D. Wang, Formation of the singularity for compressible viscoelasticity, arXiv:1109.1332.

[7] X. Huang and Z. Xin, A blowup criterion for classical solutions to the compressible NavierStokes equations, Science China-Mathematics, 53, 671-686, 2010.

[8] R.G. Larson, The Structure and Rheology of Complex Fluids, Oxford, 1995.

[9] Z. Lei, Global existence of classical solutions for some Oldroyd-B model via the incompressible limit, Chin. Ann. Math. Ser.B, 27, 565-580, 2005.

[10] Z. Lei, On 2D viscoelasticity with small strain, Arch. Ration. Mech. Anal., 198, 13-37, 2010.

[11] Z. Lei, C. Liu, and Y. Zhou, Global existence for a $2 D$ incompressible viscoelastic model with small strain, Comm. Math. Sci., 5, 595-616, 2007.

[12] Z. Lei, C. Liu, and Y. Zhou, Global solutions for incompressible viscoelastic fluids, Arch. Ration. Mech. Anal., 188, 371-398, 2008.

[13] Z. Lei, N. Masmoudi, and Y. Zhou, Remarks on the blowup criteria for Oldroyd models, J. Diff. Equ., 248, 328-341, 2010.

[14] Z. Lei and Y. Zhou, Global existence of classical solutions for the two-dimensional Oldroyd model via the incompressible limit, SIAM J. Math. Anal., 37, 797-814, 2005.

[15] Z. Lei and Y. Zhou, BKMs criterion and global weak solutions for magnetohydrodynamics with zero viscosity, Disc. Cont. Dyn. Sys. A, 25, 575-583, 2009.

[16] F. Lin, C. Liu, and P. Zhang, On hydrodynamics of viscoelastic fluids, Comm. Pure Appl. Math., 58, 1437-1471, 2005.

[17] C. Liu and N.J. Walkington, An Eulerian description of fluids containing visco-elastic particles, Arch. Ration. Mech. Anal., 159, 229-252, 2001.

[18] J. Qian and Z. Zhang, Global well-posedness for compressible viscoelastic fluids near equilibrium, Arch. Ration. Mech. Anal., 198, 835-868, 2010.

[19] Y. Sun, C. Wang, and Z. Zhang, A Beale-Kato-Majda blow-up criterion for the 3-D compressible Navier-Stokes equations, J. Math. Pure Appl., 95, 36-47, 2011.

[20] Y. Sun, C. Wang, and Z. Zhang, A Beale-Kato-Majda criterion for three dimensional compressible viscous heat-conductive flows, Arch. Ration. Mech. Anal., 201, 727-742, 2011. 